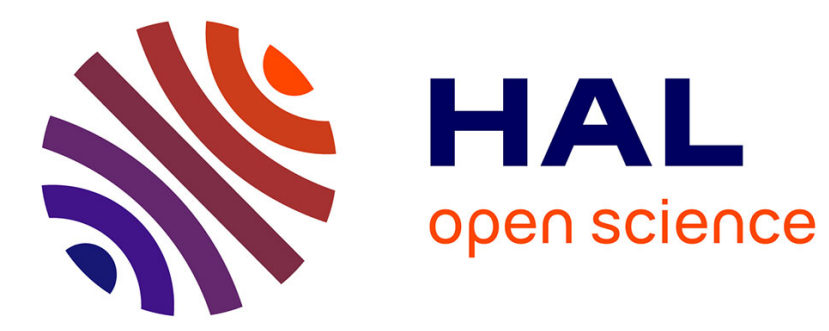

\title{
Missing data super-resolution using non-local and statistical priors
}

Ronan Fablet, François Rousseau

\section{To cite this version:}

Ronan Fablet, François Rousseau. Missing data super-resolution using non-local and statistical priors. ICIP 2015 : IEEE International Conference on Image Processing, Sep 2015, Québec, Canada. pp.676 - 680, 10.1109/ICIP.2015.7350884 . hal-01271182

\section{HAL Id: hal-01271182 \\ https://hal.science/hal-01271182}

Submitted on 8 Feb 2016

HAL is a multi-disciplinary open access archive for the deposit and dissemination of scientific research documents, whether they are published or not. The documents may come from teaching and research institutions in France or abroad, or from public or private research centers.
L'archive ouverte pluridisciplinaire HAL, est destinée au dépôt et à la diffusion de documents scientifiques de niveau recherche, publiés ou non, émanant des établissements d'enseignement et de recherche français ou étrangers, des laboratoires publics ou privés. 


\title{
MISSING DATA SUPER-RESOLUTION USING NON-LOCAL AND STATISTICAL PRIORS
}

\author{
R. Fablet, F. Rousseau \\ Institut Mines-Télécom/Télécom Bretagne; UMR 6285 LabSTICC \\ Technopôle Brest Iroise, Brest, France \\ \{ronan.fablet,francois.rousseau\}@ telecom-bretagne.eu,perso.telecom-bretagne.eu/ronanfablet
}

\begin{abstract}
We here address the super-resolution of a high-resolution image involving missing data given that a low-resolution image of the same scene is available. This is a typical issue in the remote sensing of geophysical parameters from different spaceborne sensors. Such super-resolution application involves large downscaling factor (typically from 10 to 20) and the super-resolution model should account for both texture patterns and specific statistical features, especially the spectral and non-Gaussian features. In this context, we propose a novel non-local approach and formally states the solution as the joint minimization of several projection constraints. We illustrate the relevance of the proposed model on real ocean remote sensing data, namely sea surface temperature fields, as well on visual textures.
\end{abstract}

Index Terms - super-resolution, inpainting, non-local regularisation, non-gaussian fields, spectral properties, geophysical fields, ocean remote sensing

\section{PROBLEM STATEMENT AND RELATED WORK}

Image super-resolution addresses the reconstruction or simulation of a high-resolution image given a low-resolution sample, e.g. $[7,2,6,12]$. It has received a large attention for various application domains, including multimedia application and computer graphics [7, 6], medical imaging [13], remote sensing applications $[2,5]$. These different applications may noticeably differ in the scaling to be accounted for between the low-resolution observation and the expected highresolution image. In this work, we aim at considering large scale factors up to a few tens, which are typical of the differences between the resolutions of spaceborne sensors dedicated to the space observation of the earth and the ocean.

We also assume that the high-resolution image may be partially observed, such that we aim at jointly addressing a super-resolution issue and an inpainting issue $[9,12]$. Such a super-resolution of missing data is of interest when the considered imaging sensors differ in their sensitivity to the acquisition conditions. For instance, for space oceanography, various satellites record images of the Sea surface temperature (SST), which is the temperature of the thin ocean's upper layer. Whereas Microwave (MW) radiometry provides low resolution observation (approximately a $25 \mathrm{~km}$ spatial resolution) with no effect of the cloud cover, infrared (IR) sensor delivers high resolution SST measurements (up to a few kilometers of spatial resolution) but are widely affected by the presence of clouds. Consequently, for a given region of the ocean, one may be provided with a pair of low-resolution and high-resolution SST images, the high-resolution one depicting large missing data rate.

Both non-parametric and parametric super-resolution models have been investigated. Parametric models, e.g. autoregressive models [1] and multiplicative cascade [2], provide the mean to analytically control statistical patterns, such as spectral and marginal signatures. Such models are of particular interest when explicit priors can be set, as for instance when dealing with physical processes which involve a priori known patterns (e.g., scale-invariant features). It may noted that the use of such parametric models for inpainting issues is complex and seldom explored to our knowledge. In computer vision, non-parametric pach-based schemes $[4,6,7]$ have appeared as particularly powerful methods to exploit available images or image areas as an implicit models to reconstruct new images or missing data. Such pach-based techniques may be considered both for super-resolution $[6,13]$ and inpainting [3,9] within different modeling framework, including learning-based regression [7], non-local means [13], Markov random fields $[4,6,9]$. But, to our knowledge, such patch-based schemes do not embed nor guarantee specific priors which may be available.

Here, we address the super-resolution of high-resolution images with missing data, which might also be regarded as multi-resolution inpainting. From a methodological point of view, we introduce a non-local patch-based setting, which also embeds additional statistical priors, namely priors on the high-resolution detail and on the radial covariance structure. Formally, the super-resolution is stated as the joint minimization of different energy terms to fulfill all constraints and priors. We demonstrate and illustrate the relevance of this model for both visual textures and geophysical fields (here, SST fields) with a scaling factor of 16 . The subsequent is organized as follows. Section 2 first briefly introduce non-local priors for inverse problems in image processing. We detail in 
Section 3 the proposed model and its numerical resolution. Numerical experiments on real data are reported in Section 2 along with concluding remarks.

\section{EXEMPLAR-BASED INPAINTING AND NON-LOCAL REGULARISATION}

Patch-based techniques are particularly appealing to reconstruct and simulate textured images from previous observations $[4,6,13,9]$. In this context, non-local regularisation priors introduce an energy minimisation and inverse image problems are stated as the minimisation of a global energy[11]:

$$
\widehat{I}=\arg \min _{I} U_{o b s}\left(I, I_{\text {obs }}\right)+\gamma U_{N L R e g}(I)
$$

In the simplest cases, observation term $U_{o b s}\left(I, I_{o b s}\right)$ resorts to a quadratic error term $\left\|I-I_{o b s}\right\|^{2}$. Non-local prior $U_{N L R e g}(I)$ involves the reconstruction of image $I$ from a reference patch database, where a patch is a $N \times N$ local window of an image with $\mathrm{N}$ typically ranging from 3 to 15 . Let us denote by $\left\{\mathcal{A}_{k}\right\}_{k \in \mathcal{K}}$ the colected set of representative image patches, and $p_{0}$ the pixel referring to the center of the patch. Following [13], non-local (NL)prior $U_{N L R e g}(I)$ is stated as:

$$
U_{N L R e g}(I)=\left\|I-\mathcal{P}_{N L M}\left(I,\left\{\mathcal{A}_{k}\right\}\right)\right\|^{2}
$$

where $\mathcal{P}_{N L M}\left(I,\left\{\mathcal{A}_{k}\right\}\right)$ is the non-local projection of image $I$ onto patch database $\left\{\mathcal{A}_{k}\right\}$ given by a non-local mean [13]. For a given pixel $p$, the non-local projection of the patch $\mathcal{V}_{p}$ around $p$ is given by $\forall q \in \mathcal{V}_{p}$,

$$
\mathcal{P}_{N L M}\left(I,\left\{\mathcal{A}_{k}\right\}\right)(q)=\sum_{k \in \mathcal{K}} w_{k}\left(I, \mathcal{A}_{k}, p\right) \mathcal{A}_{k}(q-p)
$$

where $\mathcal{V}_{p}$ refers to the $N \times N$ pixel in the patch around $p$ and $\mathcal{A}_{k}(s)$ to the value of patch $\mathcal{A}_{k}$ at site $s$. $w_{k}\left(I, \mathcal{A}_{k}, p\right)$ is the normalised weight stating the contribution of patch $\mathcal{A}_{k}$ to the projection of image $I$ at pixel $p$. In the classical nonlocal-means approach $[11,13]$, it is stated as : $w_{k}\left(I, \mathcal{A}_{k}, p\right) \propto$ $\exp \left[-\eta \cdot d\left(I, \mathcal{A}_{k}, p\right)\right]$ with $d\left(I, \mathcal{A}_{k}, p\right)$ the mean square difference between reference patch $\mathcal{A}_{k}$ and the patch of size $N \times N$ around $p$ in image $I . \nu$ is a model parameter. This setting also includes nearest-neighbor settings, for an infinite $\nu$ value. Non-local setting may also differ in the combination of the projected image patches to reconstruct an image. One may apply the projection to each image patch and consider for the NL image only the values at the center of the projected patches. One may also apply the projection to a subsample of image pixels and consider the entire projected patches to compute the NL image, as in patch-based texture resampling technique $[6,3]$.

\section{PROPOSED NON-LOCAL MODEL}

Here, we investigate a non-local model for the super-resolution of missing data in high-resolution observation of the sea sur- face such that:

- the low-resolution projection of the reconstructed image $I$, denoted as $\mathcal{P}_{L R}[I]$ should conform to the observed low-resolution image $I_{L R}^{o b s}$. In this work, we consider numerical experiments for $2^{S}$ scaling factor and the low-resolution projection is assumed to be provided by a wavelet decomposition, where all detail coefficients up to level $S$ are set to 0 ;

- the reconstructed image $I$ should conform to the highresolution image $I_{H R}^{o b s}$ for some actually observed image domain $\Omega$.

Besides, we consider additional priors to account for specific statistical image features, such as specific non-Gaussian and covariance patterns. Formally, we resort to stating the following additional constraints:

- the reconstructed image $I$ should depict a reference radial covariance structure given by radial power spectral density $r P S D^{*}$;

- the high-resolution detail $I-\mathcal{P}_{L R}[I]$ should conform to an expected marginal distribution.

Both reference radial power spectral density $P S F^{*}$ and $\mathcal{D}_{\theta}$ might be set a priori or inferred from the observed data using both parametric and/or non-parametric models. In the reported experiments, we consider here parametric models for $\mathcal{D}_{\theta}$, namely generalized Gaussian models to account for non-Gaussian fields. This prior is fitted to available highresolution data. For the prior on the covariance structure, we consider a non-parametric model issued from an empirical estimation of radial covariance structure. Other parametric models and non-parametric models may be considered without any loss of generality.

Given these priors, we formally resort a non-local setting and state the super-resolution of missing data as a constrained minimization issue as follows:

$$
\begin{aligned}
& I_{H R}=\arg \min _{I}\left\|I-\mathcal{P}_{N L M}\left(I,\left\{\mathcal{A}_{k}\right\}\right)\right\|^{2} \\
& \text { Subject to }\left\{\begin{array}{l}
I_{H R}(\Omega)=I_{H R}^{o b s}(\Omega) \\
\mathcal{P}_{L R}[I]=I_{L R}^{o b s} \\
I(p)-I_{L R}(p) \propto \mathcal{G} \mathcal{G}_{\sigma, \alpha} \\
r P S D(I)(\omega)=r P S D^{*}(\|\omega\|)
\end{array}\right.
\end{aligned}
$$

where $\mathcal{P}_{N L M}(I)$ refers to the non-local projection of image $I$ given by (Eq.3), $\mathcal{G G}_{\sigma, \alpha}$ the centered generalised Gaussian distribution fitted to the marginal distribution of the observed high-resolution detail with scale parameter $\sigma$ and exponent $\nu$.

Regarding the numerical resolution of the above constrained minimization, it might be noted that all terms and 

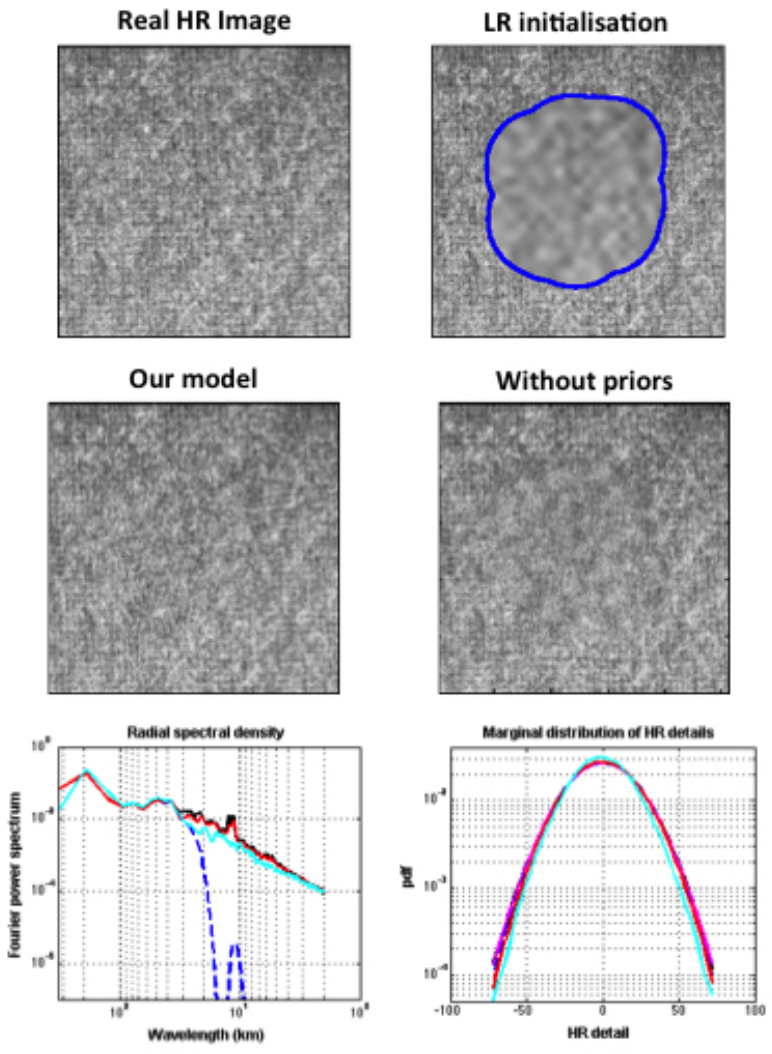

Fig. 1. Super-resolution of missing data for a visual texture: first row, real high-resolution (HR) image, initial interpolation using the low-resolution image, interpolated image using the proposed non-local model with and without the priors on the HR details and on radial covariance structure; second row, radial spectral densities of the real HR image (black,-), the real LR image (blue, -) and the non-local reconstruction with (red,-) and without (cyan, -) the priors on the HR details and on radial covariance structure, multifractal spectrum, marginal distribution of the HR details.

constraints can be regarded as projections onto image subspaces. Especially, the constraint on the marginal distribution of the high-resolution details can be solved for using a contrast change to fit the expected marginals, and the spectral constraint resorts to an isotropic filtering, performed in the Fourier domain. For the projection to the low-resolution subspace, we apply a wavelet-based projection assuming a $2^{S}$ downscaling factor. For the non-local projection, we randomly select a sequence of non-overlapping image patches. The non-local reconstruction for a given image patch amounts to replacing the image patches by their non-local version (3) for the interior of each patch (we typically use a 3-pixel wide boundary). Overall, the proposed numerical scheme then iterates the following three main steps : i) non-local projection, ii) contrast change onto the the high-resolution details to fit the expected marginal distribution, iii) Fourier-based filtering to match the expected spectral law. Each step also involves the projection onto the low-resolution and interpolation subspace. As initialization, we consider the projection of a null or white noise image onto the low-resolution and interpolation constraints. Our model involves two key parameters: the patch size $N \times N$ and scale parameter $\beta$ for the non-local projection $\mathcal{P}_{N L M}\left(\cdot,\left\{\mathcal{A}_{k}\right\}\right)$. In the reported results, we use $N=15$ and $\eta=\infty$ (i.e., nearest-neighbor search in (3)). As similarity measure between image patches, we combine the mean squared differences between the image patches at highand low-resolution.

\section{RESULTS}

We report numerical experiments for for a visual texture (Fig.1) and a satellite SST observation (Fig.2). In each case, we simulate an associated low-resolution observation using a 16-downsampling factor and a Daubechies-6 low-pass filter. We randomly generate a missing data mask accounting for about $20 \%$ of the image area. As patch database, we randomy select 5000 image patches from high-resolution image patches with no missing data. Empirically, we noted that the iterated projection step converge rapidly. All the reported results involve five iterations of the four main projection steps described above.

The reported examples demonstrate that we reconstruct consistent high-resolution images which actually jointly account for four key features: a low-resolution component which conforms to the low-resolution observation, highresolution information provided by the high-resolution data if available, the expected patterns of the radial power spectral density and of the marginal distribution of the high-resolution details. To our knowledge, no existing state-of-the-art models can jointly fulfil these four constraints. Visually, the differences are more important for the SST image. Still, the additional prior on the covariance structure slightly improves the reconstruction of the square-like pattern of the visual texture. Regarding the SST example, the direct application of the non-local patch-based model actually depict a relevant marginal distribution of the high-resolution detail, but involve two much fine-scale energy as illustrated by the reported radial power spectral densities. This results in a reconstructed image which depicts two many fine-scale details. By contrast, the superimposed level-lines show that the proposed model resort to a relevant interpolation, which depicts visually consistent geometrical patterns.

Our results demonstrate that, using a non-local regularization setting, we can combine implicit patch-based image priors to explicit statistical priors on image features, here the power spectral density and the marginals of the highresolution details. This is of key interest for the develpment of non-local/patch-based techniques in physical and geophysical imaging applications, which typically involve priors on the underlying processes. Future work will further explore 

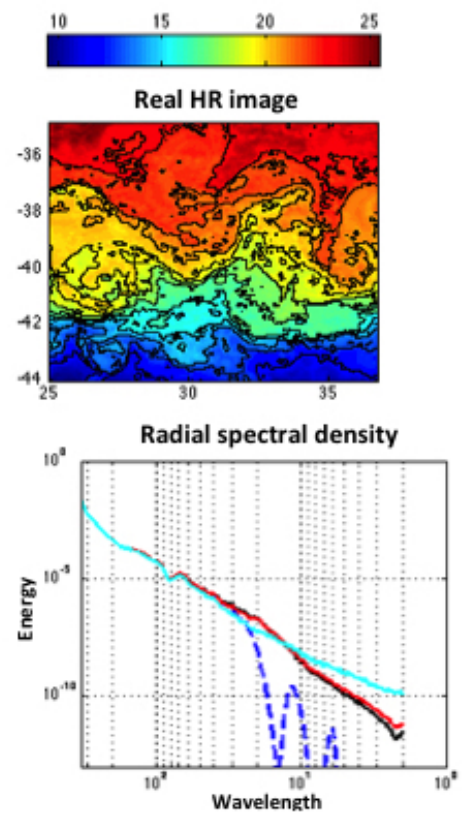

Pdf of HR details

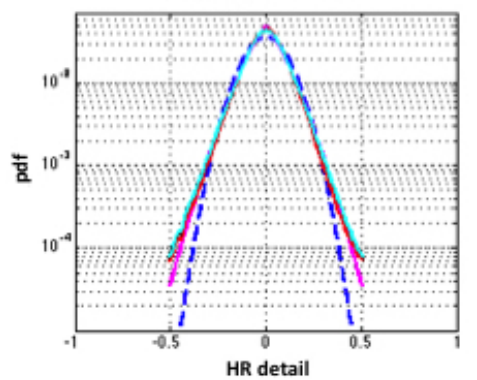

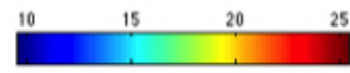

LR Initalisation
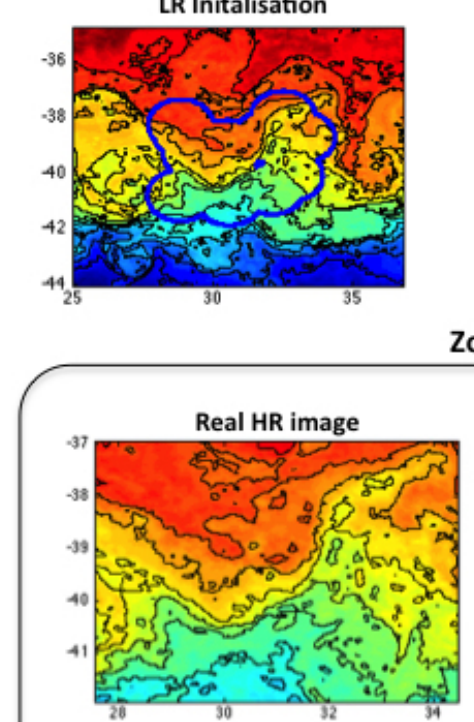

Zoom

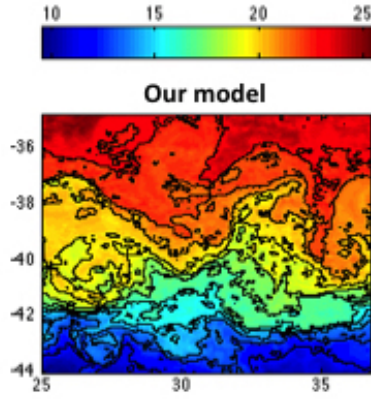

西
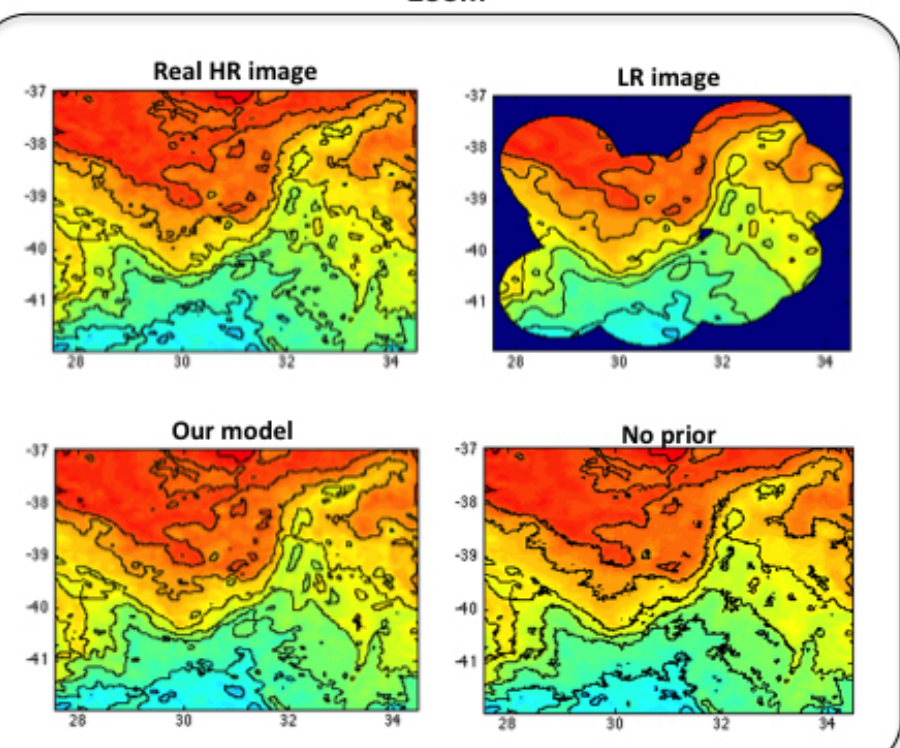

Fig. 2. Super-resolution of missing data for a high-resolution SST field: first row, real high-resolution (HR) image, initial interpolation using the low-resolution image, interpolated image using the proposed model; bottom left, radial spectral densities of the real HR image (black,-), the real LR image (blue,-) and the reconstructed image using our model with (red,-) and without (cyan, -) priors on the high-resolution details and on the spatial covariance, marginal distribution of the HR details; bottom right zoom on the missing data area for the real SST image, the low-resolution image, the reconstructions using our non-local model with and without the additional priors. We superimpose image level-lines to illustrate the underlying spatial geometry.

time and space-time extension of the proposed model for super-resolution and inpainting issues in multi-sensor image time series.

\section{Acknowledgements}

This work was supported by ANR (Agence Nationale de la Recherche, grant ANR-13-MONU-0014) and "Fondation Télécom" (grant "Futurs \& Ruptures" A2Ocean).

\section{REFERENCES}

[1] Byung Tae Oh, Jay Kuo, C.-C. Super-resolution texture synthesis using stochastic PAR/NL model. JVCR, 23(7):995-1007, 2012.
[2] Chainais, P. König, E., Delouille, V., Hochedez, J.-F. Virtual super resolution of textured images using multifractal stochastic processes. JMIV, 39(1):28-44, 2011.

[3] Criminisi, A., Perez, P., Toyama, K. Region filling and object removal by exemplar-based image inpainting. IEEE Trans. on Image Processing, 13(9):1200-1212, 2004.

[4] Efros, A.A., Freeman, W.T. Image Quilting for Texture Synthesis and Transfer. Proc. ACM SIGGRAPH, 2001.

[5] Fablet, R., Boussidi, B., Autret, E., Chapron B. Random walk models for geometry-driven image super-resolution. Proc. 38th Int. Conf. on Acoustics, Speech and Signal Processing, ICASSP'2013, 2013.

[6] Freeman, W.T., Liu, C. Markov Random Fields for Superresolution and Texture Synthesis. In A. Blake, P. Kohli, and C. Rother, eds., Advances in Markov Random Fields for Vision and Image Processing, Chapter 10. MIT Press, 2011. 
[7] Glasner, D., Bagon, S., Irani, M. Super-resolution from a single image. in IEEE Int. Conf. on Computer Vision, CCV'2009, 2009.

[8] He, H., Siu, W. C. Single image super-resolution using Gaussian process regression. In IEEE Conf. on Computer Vision and Pattern Recognition, CVPR'2011, 449-456.

[9] Le Meur, O., Ebdelli, M., Guillemot, C. Hierarchical SuperResolution-Based Inpainting. in IEEE Trans. on Image Processing, 22(10):3779-3790, 2013.

[10] Lguensat, R., Tandeo, P., Fablet, R., Garello, R. Spatiotemporal interpolation of Sea Surface Temperature using high resolution remote sensing data. Proc. IEEE OCEANS'2014, St. John's, Canada, sept. 2014.

[11] Peyré, G., Bougleux, S., Cohen, L. Non-local Regularization of Inverse Problems. Proc. Eur. Conference on Computer Vision, ECCV'2008, 57-68, 2008.

[12] Romano, Y., Protter, M., Elad, M. Single Image Interpolation Via Adaptive Nonlocal Sparsity-Based Modeling. IEEE Trans. on Image Processing, 23(7):3085-3098, 2014.

[13] Rousseau, F. A non-local approach for image super-resolution using intermodality priors. Medical Image Analysis, 14(4):594$605,2010$. 